\title{
The Importance of Standardizing Acquisition Settings and Interpretation Criteria of Radionuclide Cisternography
}

\author{
Francesco Cicone $^{1,2, *} ;$ Carolina Del Mastro $^{1}$; Francesco Scopinaro ${ }^{1,2}$ \\ ${ }^{1}$ Unit of Nuclear Medicine, Sant' Andrea Hospital, Rome, Italy \\ ${ }^{2}$ Department of Surgical and Medical Sciences and Translational Medicine, Sapienza University of Rome, Rome, Italy \\ ${ }^{*}$ Corresponding author: Francesco Cicone, Department of Surgical and Medical Sciences and Translational Medicine, Sant' Andrea Hospital, Sapienza University of Rome, Rome, Italy. \\ E-mail: f.cicone@iol.it
}

Received: November 12, 2012; Revised: February 16, 2013; Accepted: March 11, 2013

Keywords: Radionuclide Cisternography; Cerebrospinal Fluid Leakage; Spontaneous Intracranial Hypotension; CSF

\section{Dear Editor}

We read with interest the paper by Lu and colleagues on radionuclide cisternography (RNC) entitled "The Value of Changing Position in the Detection of CSF Leakage in Spontaneous Intracranial Hypotension Using Tc-99m DTPA Scintigraphy: Two Case Reports", claiming an additive value of scanning the patients in the upright position for the detection of cerebrospinal fluid(CSF) leakage (1). In two cases of spontaneous intracranial hypotension (SIH), the authors observed the appearance of multiple images of increased radioactivity on both sides of the spinal canal after changing the patient position, which were interpreted as direct signs of CSF leaks.

We fully agree with Lu and colleagues that direct signs of leakage should be always looked for in RNC, as early appearance of bladder radioactivity is an unreliable indirect sign, probably due to traumatism after lumbar puncture. This was elegantly shown by Takahashi et al. (2) and subsequently confirmed by others. However, in our opinion, the images of increased radioactivity showed by Lu et al. symmetrically distributed on both sides of the spinal canal, should not be regarded as direct signs of leakage. Rather, these images might correspond to prominent structural abnormalities such as meningeal diverticula or nerve root cysts. These anatomical variants, together with any other structural meningeal weaknesses, are known to confer an increased risk of CSF leaks, potentially requiring corrective surgery (3). When the patient stands up, CSF is likely to spread to the loci of least resistance, not seen in the supine position. Correlative radiological imaging would be needed to show underlying anatomical abnormalities. In Figures 1 and 2 we give an example of that.

$\mathrm{SIH}$ is being more frequently recognized as an important cause of persistent headache with rather typical clinical presentation (3). Symptom relief after epidural blood patching often provides a "ex-juvantibus" diagnosis. Although MRI and CT-myelography have nowadays the greatest impact on the diagnostic workout, being able to accurately define the extent and location of CSF leaks. However, RNC may still play a role in selected, uncertain cases. Unfortunately, a limitation of RNC is represented by the lack of a standardized acquisition protocol. Some authors suggested to acquire multiple early images in the lateral position after intrathecal injection (2), while our group and others have favored a change of posture for detecting CSF leaks with increased sensitivity $(4,5)$. Image quality and the choice of radionuclide represent additional issues, as signs of extradural activity accumulation are often subtle and difficult to detect.

In conclusion, we think that the paper by Lu et al. provides a valuable suggestion for improving the RNC acquisition technique. However, larger case series are needed to prove whether changing the patient's position does really offer a diagnostic advantage in terms of direct leakage detection. Moreover, comparative imaging would also be warranted in order to standardize the interpretation criteria of RNC, providing a compelling definition of its current role in the diagnosis of CSF leakage.

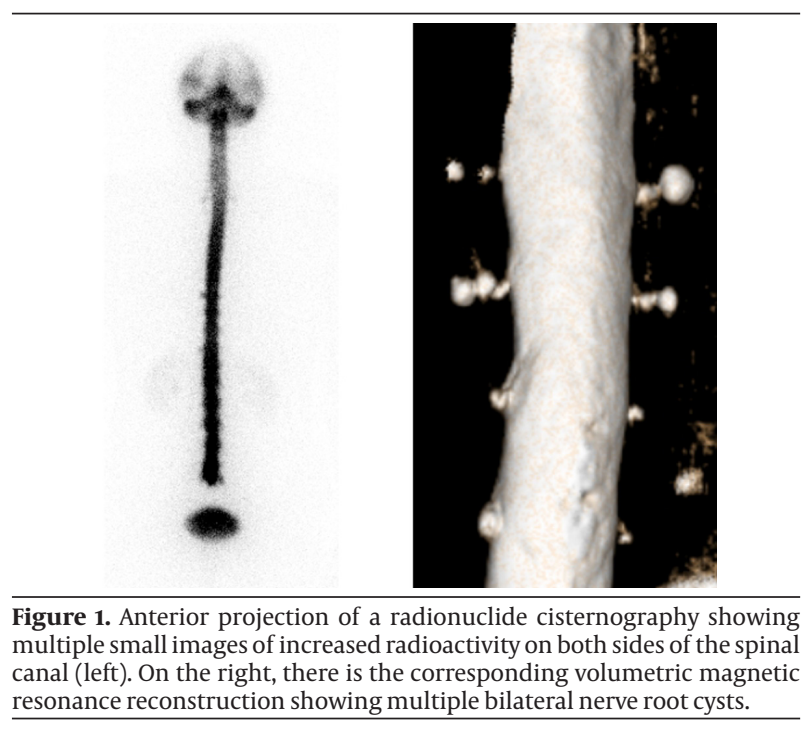

Copyright (C) 2014, Tehran University of Medical Sciences and Iranian Society of Radiology. This is an open-access article distributed under the terms of the Creative Commons Attribution-NonCommercial 4.0 International License (http://creativecommons.org/licenses/by-nc/4.0/) which permits copy and redistribute the material just in noncommercial usages, provided the original work is properly cited. 


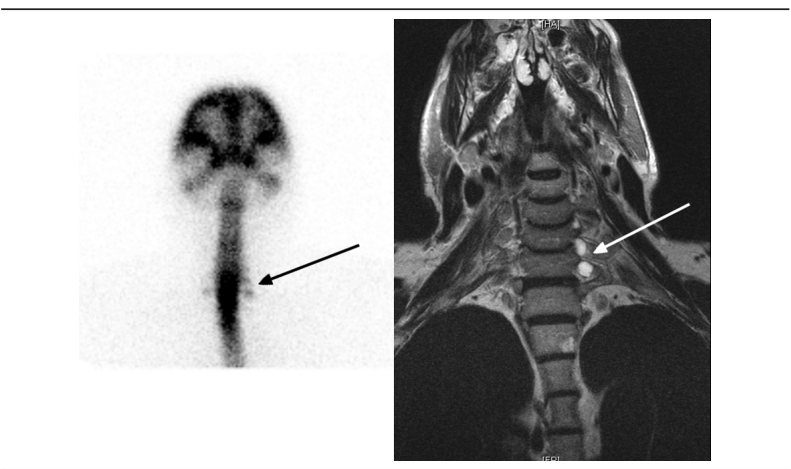

Figure 2. The spine at the cervico-thoracic level in the same patient. Nerve root cysts (arrows) are seen on both radionuclide cisternography (left) and magnetic resonance imaging (right).

\section{References}

1. Lu YY, Wang HY, Lin Y, Lin WY. The Value of Changing Position in the Detection of CSF Leakage in Spontaneous Intracranial Hypotension Using Tc-99m DTPA Scintigraphy: Two Case Reports. Iran J Radiol. 2012;9(3):150-3.

2. Takahashi M, Momose T, Kameyama M, Mizuno S, Kumakura Y, Ohtomo K. Detection of cerebrospinal fluid leakage in intracranial hypotension with radionuclide cisternography and blood activity monitoring. Ann Nucl Med. 2005;19(4):339-43.

3. Schievink WI. Spontaneous spinal cerebrospinal fluid leaks and intracranial hypotension. JAMA. 2006;295(19):2286-96.

4. Inenaga C, Tanaka T, Sakai N, Nishizawa S. Diagnostic and surgical strategies for intractable spontaneous intracranial hypotension. Case report. J Neurosurg. 2001;94(4):642-5.

5. Del Mastro C, Cicone F, D'Andrea G, Scopinaro F. Tc-99m DTPA cisternography shows disappearance of cerebrospinal fluid leakage after standing a short time. Clin Nucl Med. 2009;34(1):29-30. 